\title{
E-MRS/UUMRS-ICEM 2006 Spring Meeting Emphasizes Functional Materials and Devices
}

The 2006 European Materials Research Society Spring Meeting was held in conjunction with the International Union of Materials Research Societies International Conference on Electronic Materials (EMRS/IUMRS-ICEM 2006) in Nice, France, May 29-June 2, 2006. Functional materials and devices served as a common thread running through the 23 technical symposia, which were organized into six clusters: Nanomaterials and Nanoelectronics, Synthesis and Characterization of Functional Materials, Materials for Energy, Composite and Organic Materials, Semiconductor Materials, and Education and Tutorials. Research conducted at the nanoscale was also prominent in most of the symposia. More than 2,500 attendees from 62 countries participated in the fiveday conference. A plenary session, poster sessions, an equipment exhibit, and an awards ceremony were additional highlights of the event. The meeting chairs were Abdelillah Slaoui (Centre National de la Recherche Scientifique, Strasbourg, France), Rodrigo Martins (Universidade Nova de Lisboa, Portugal), Peter Glasow (E-MRS, Germany), and Lian Zhou (Chinese Academy of Science, Beijing, China).

\section{Plenary Session}

The plenary session offered four major speakers who each incorporated the concept of nanoscience and nanotechnology in their presentations. In the first lecture, on energy, Mildred S. Dresselhaus of the Massachusetts Institute of Technology said, "Electricity was not discovered via incremental improvements to the candle." She said new science and new technology are needed to make quantum leaps in the field, which she discussed in her talk, "Addressing Grand Energy Challenges through Nanoscience." The rapid economic growth of countries such as China, Brazil, and India translates to an increasing demand for energy. Dresselhaus said that the global energy demand, currently at 14 TW, is anticipated to rise to $60 \mathrm{TW}$ by 2050. Nanomaterials and nanotechnology, she said, have enormous potential to address this energy crisis. For example, she said, research in nanomaterials has influenced tremendous strides in the area of solid-state lighting with its promise of enhanced efficiency and energy savings. Nanotechnology also plays a role in the development of new materials for hydrogen storage, novel catalysts, and innovative production techniques for the applica-

www.emrs-strasbourg.com

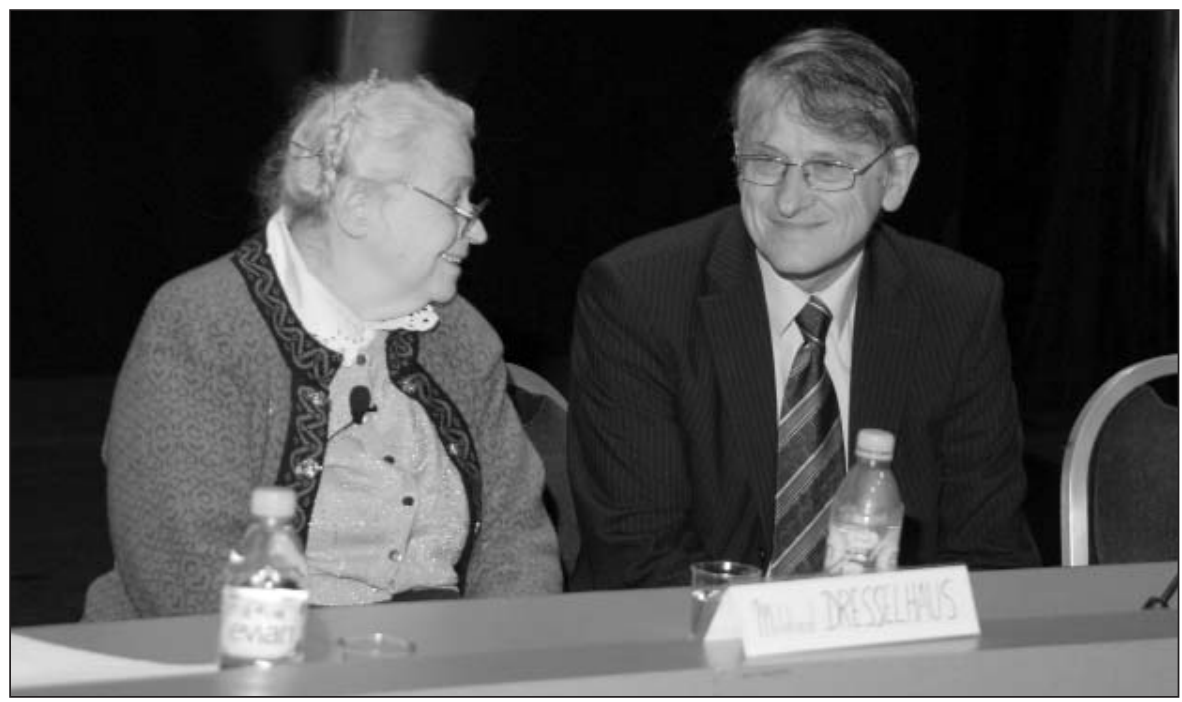

Plenary speakers Mildred S. Dresselhaus of the Massachusetts Institute of Technology (left) and Klaus von Klitzing of the Max Planck Institute for Solid-State Research shared a few moments before the session begins at the E-MRS/IUMRS-ICEM 2006 Meeting in Nice, France, held May 29-June 2, 2006.

tion of hydrogen in addressing energy needs, Dresselhaus said.

In a separate plenary address focusing on energy, Martin Green of the University of New South Wales, Australia, said that solar energy has seen a resurgence in the last few years and could become the solution to the world's energy problems over the next several decades. The currently installed photovoltaic cells around the world are first-generation and based on silicon wafers, he said. The second generation includes various thin films that are at present partially in use and partially under research and development. These include amorphous, micro-, and nanocrystalline Si thin films; chalcogenides such as CdTe and CIGS (thin films, typically, of copper indium gallium diselenide); and dye-sensitized and organic thin films. Third-generation photovoltaics represent the future of the technology, said Green, offering higher efficiencies and durability at a lower cost and using abundantly available nontoxic materials. He said that nanomaterials will also play a significant role in the development of third-generation photovoltaic applications. Some of the possibilities for third-generation photovoltaics include stacking of thin films for greater efficiency, tandems, quantum dots, and improved dye-sensitized and organic thin films.
"Research is the transformation of money to knowledge. Innovation is the transformation of knowledge to money," said plenary speaker Hans Meixner of Siemens AG, Germany, in his presentation on the piezo effect and its influence on the development of a new generation of actuators. Showing the present design that is universally used in its basic form, Meixner said that the latest generation of injectors, which use the new actuators, has significantly helped increase fuel efficiency by $50 \%$ or more in gasoline engines, thus aiding energy conservation efforts and reducing automobile exhaust and carbon-particle pollution in the atmosphere. In describing the materials properties of the piezoelectric materials used, Meixner talked about the sintering of the ceramics and the use of multilayer stacks of the material that were critical to the development process.

Plenary speaker Klaus von Klitzing of the Max Planck Institute for Solid-State Research received the 1985 Nobel Prize in physics for the discovery of the quantized Hall effect. In his presentation at the meeting, von Klitzing focused on possible applications of the quantum Hall effect in materials research. Among the examples he gave are a dc step-up transformer based on a bilayer quantum Hall system and an unexpected phenomenon 
for quantum Hall systems under microwave radiation. Von Klitzing also discussed quantum dots, which may be key for quantum computers.

\section{Nanomaterials and Nanoelectronics}

In Symposium A on current trends in nanoscience, D. Bonnell (Univ. of Pennsylvania, USA) listed about 20 properties of nanostructures that can be measured using various techniques such as atomic force microscopy (AFM). She also introduced a method called ferroelectric nanolithography, wherein electric fields can be written by multiple-modulation scanning probe microscopy (SPM) on a substrate which is then used as a template for the formation of aligned nanostructures. In Symposium $\mathrm{F}$ on nanoscale imaging and metrology of devices and innovative materials, R. Sharma (Arizona State Univ., USA) described the environmental transmission electron microscope (ETEM), an instrument modified so that it can be used to follow gas-solid and/or liquid-solid interactions at elevated temperatures. Although the history and development of the ETEM is as old as the TEM itself, developments in the design of mediumvoltage microscopes have reduced resolutions down to the subnanometer level. The ETEM in Sharma's laboratory includes an environmental chamber surrounding the specimen. The chamber incorporates a differential pumping system such that the pressure within could be in the $\sim 10$ Torr range while the pressure outside, within the TEM column, is in the typical $10^{-10}$ Torr range, similar to a regular TEM. Thus, there is no effect on the performance of the microscope due to the presence of the environmental chamber.

S. Takagi (MIRAI-AIST, Japan; Univ. of Tokyo, Japan) emphasized in Symposium $\mathrm{B}$, addressing strained silicon, that mobility-enhancement technologies are mandatory for future scaled metal oxide semiconductor field-effect transistors (MOSFETs), and described results on high hole-mobility $p$-type MOSFETs using $\mathrm{SiGe} / \mathrm{Ge}$ channels with and without strain. He used both SiGe/Ge substrates and $\mathrm{SiGe/Ge}$ channel regions grown locally on Si wafers. These latter devices have shown hole mobilities as high as 10 times that of bulk silicon. In short-channel (40 nm) MOSFETS, the drain current is increased by $80 \%$, demonstrating that the high field mobility may also be increased.

For devices whose gate lengths are $35 \mathrm{~nm}$ or less, strained silicon represents an improvement in performance, said S. Thomson (Univ. of Florida, USA) in Symposium B. This improves the mobility of holes and electrons in the material; a stress of 3 GPa can result in a $35 \%$ improvement in $n$-type MOS and $90 \%$ improvement in $p$-type MOS, Thomson said. The real limiter to scaling now is not the gate length but the external resistance to the device, and future devices will need to have increased carrier mobilities, he said.

S. Koester (IBM, USA) said that III-V compounds are becoming increasingly important, as the scaling of silicon devices is slowing down. Strained silicon can be used to improve $p$-type FETs, but $n$-type FETs require compounds such as gallium arsenide. He described, in Symposium B, experiments in making MOSFETs in gallium arsenide using hafnium oxide and aluminum oxide. Inserting $\mathrm{Si} / \mathrm{SiO}_{2}$ interlayers improved the density of states at the surface by an order of magnitude.

Nanoscale properties such as carrier confinement, Coulomb blockade, and increased bandgap would be very important if they could be applied to silicon. In Symposium E on nanowires and nanotubes, J. Arbiol (Univ. of Barcelona, Spain) described the growth and structure of silicon nanowires on amorphous silica using gold and copper as catalysts in a chemical vapor deposition (CVD) reactor. At low temperature $\left(\sim 500^{\circ} \mathrm{C}\right)$, the wires grew straight without any gold or copper contamination. At higher temperatures, the growth was erratic and the wires were covered with metal particles. TEM results and models demonstrated that using $\mathrm{Au}$ as the catalyst leads to silicon nanowires with [103], [101], and [110] orientations, while copper causes [001] growth.

\section{Functional Materials}

Nanowires and nanotubes have been previously used for sensing applications using electrically driven sensors that function by an absorbate-mediated conductivity mechanism. P. Yang (Univ. of California, Berkeley, USA) and his group, however, have focused on the excellent waveguiding abilities of specific highrefractive-index nanowires to create subwavelength evanescent-wave sensors that take advantage of the optical properties of the materials. In Symposium G on functional materials for micro- and nanosystems, Yang demonstrated waveguiding in nanowires in liquids-including glycol and water-that is affected by the presence of chemicals, thereby leading to sensing capabilities. He described how such waveguides can be integrated into microfluidic devices as well as lead to applications such as detecting single DNA molecules. The major advantages of the technique are the high sensitivity achieved and the specific chemical signatures detected.

In a poster presentation for Symposium $\mathrm{H}$ on photon-assisted synthesis and processing of functional materials, J.M. Fernández-Pradas (Univ. of Barcelona, Spain) described the use of a Nd:YAG laser beam as a tool to print patterns of colored enamels on tile substrates. The laser beam is scanned over a layer of enamel powder previously deposited on the tile surface. The laser energy can be focused to heat a small zone without affecting the rest of the piece, compared with traditional furnace techniques in which the whole piece has to be heated. Fernández-Pradas presented a study of the interaction between a green enamel based on chromium oxide pigment and the white tile substrate after laser irradiation. Lines obtained with different combinations of laser beam power and scan speeds were investigated. The characteristics of the marked lines were found to be directly related to the energy density used during irradiation. A linear relationship was observed between the energy density and the volume of substrate material affected. Optimum results were obtained when energy density was sufficient to only melt the enamel and a shallow zone of the substrate.

Significant performance improvements have been made in poly-Si thin-film transistors (TFTs) after the wide acceptance of laser-based lateral crystallization technology and its numerous variants. However, variations in TFT characteristics still present substantial difficulties. In his presentation in Symposium I on thin films for large-area electronics, M. Crowder (Sharp Labs, USA) discussed results on a study of the characteristics of poly-Si TFTs fabricated by laser crystallization and their relationship to the microstructural details of the active layer in the TFTs. The effects of single grain-boundary inclusions in the device channel were discussed, and the key causes of variation in performance were described even in the absence of hard boundaries. The existence of structural defects and variations in texture correlated well with the observed variation in TFT mobility and threshold voltage. Texture here refers to the dominant crystallographic orientation with the region corresponding to the device active layer. The study demonstrates the importance of achieving a consistent microstructure as well as the difficulty in doing so by employing blanket crystallization technologies.

\section{Materials for Energy}

Hydrogen has potential as a clean energy source for fuel cell 
applications in the future. An energy density 3000 times that of gasoline is required for hydrogen. In his presentation in Symposium M on sustainable energies, E. Akiba (AIST, Japan) made the case for using magnesium in the form of magnesium hydride as an efficient hydrogen storage material. The hydrogen capacity of this material is 7.6 mass \%, and the storage can be reversed for generating hydrogen even though the reaction temperature is about $600 \mathrm{~K}$. Akiba said that the use of Mg-based bcc alloys could help reduce this reaction temperature. His group used a ball-milling technique for lower-temperature synthesis of a Mg-Ti-based alloy. However, this material did not absorb hydrogen, due to its large lattice parameter of $0.34 \mathrm{~nm}$. Therefore, it was decided to use Co instead of $\mathrm{Ti}$, and a $\mathrm{Mg}_{50} \mathrm{Co}_{50}$ alloy, with a lattice parameter of $0.3199 \mathrm{~nm}$ and a maximum hydrogen capacity of $2.1 \mathrm{wt} \%$, was developed using the ball-milling technique. The study showed that ball milling is indeed an effective technique for synthesizing $\mathrm{Mg}$-based alloys for hydrogen storage applications and that the lattice parameter of the bcc structure is an effective guideline.

Symposium $\mathrm{O}$ held a forum to discuss Solarpact (Solar Power from Advanced Cadmium Telluride), which is a technical network connecting relevant research groups and industrial units from the United States and Europe in a targeted approach to promote the technology of photovoltaic (PV) modules based on the semiconductor cadmium telluride and related compounds for cost-competitive large-scale mass production. Midterm goals for the group are a $15 \%$ efficiency at a cost of 1 euro per peak watt, with longterm goals of better than $18 \%$ efficiency at a cost of 0.5 euros per peak watt by the year 2020. P. Meyers gave a report on his company, First Solar, which has produced $36 \mathrm{MW}$ modules to date, most of which are sold in Germany.

CdTe is made from zinc mining waste, and its manufacture is environmentally friendly. More than three times the cadmium is released in the equivalent production of silicon as is released in cadmium telluride production. In fact, much more cadmium is released into the atmosphere from the burning of oil than from CdTe production, according to $\mathrm{V}$. Fthenakis (Brookhaven Natl. Lab, USA). Meyers said that Solarpact should formulate a statement of objectives, including one good model for the devices, covering such topics as evaluation measurements, materials science, film growth, and device architecture.

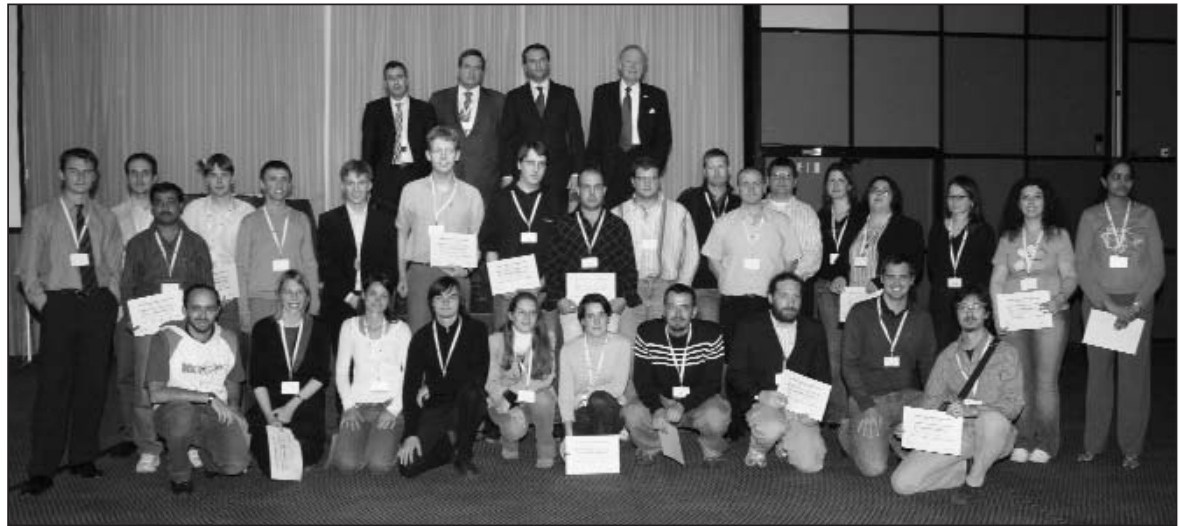

Graduate students received awards in the presence of conference organizers and the president of E-MRS.

In Symposium $\mathrm{N}$ on nuclear materials and materials for fusion, L. Mansur (Oak Ridge Natl. Lab, USA) gave an update on the construction of the spallation neutron source (SNS) at Oak Ridge. The first neutron beam was produced in April of this year at the facility. The SNS is a type of high-power accelerator, similar to ionbeam and particle physics accelerators. Mansur said that expertise accumulated over the last few decades with fission and fusion reactors has now been used for the SNS design. There are several radiationdamage-induced property changes to consider for materials used in the accelerators and reactors, the major ones being embrittlement, swelling, and radiation creep. Mansur discussed how 316LN stainless steel was eventually chosen as the material to be used for the SNS target area which contains mercury. In addition to the central issue of radiation effects in metallic structural alloys, designers were concerned with radiation effects in polymers and ceramics, which could be employed as seals, sensors, or insulators.

\section{Composite and Organic Materials}

The overall topic of composite and organic materials consisted of two symposia: solid-state ionics (Symposium P) and chemical and biosensing transistors (Symposium Q). Discussions in Symposium $\mathrm{P}$ showed that solid-state ionics is a dynamic and still-growing field of research concerning the transformation and storage of energy by batteries and fuel cells, chemical sensors and actuators, new principles of information storage as well as general materials properties such as transport of ions and electrons, thermodynamic stability, use of materials in catalysis, and electronics.

In Symposium Q, R. Duran (Univ. of
Florida, USA) introduced singlechannel-level measurements of a new sensor device that combines the functional properties of silicon-based microelectronic devices with those of genetically expressed ion channels. The ion channel is one of nature's most sensitive switching elements, simultaneously delivering intrinsic amplification of 10,000 or more in a single stage. Duran's group was able to interface several different ion channels to gate surfaces with the eventual goal of exploiting analyteinduced changes in single-channel activity for sensor applications. The device was shown to operate in voltage-ramped, acstepped, or constant-voltage mode.

\section{Semiconductor Materials}

In Symposium U on Si-Based Materials for Advanced Microelectronic Devices, N. Cowern (Univ. of Surrey, UK) presented a theory describing how the percentage activation of boron implanted in silicon can be improved to close to $100 \%$ by paying attention to the role of vacancy production (vacancy engineering). Silicon ions are co-implanted with boron at such an energy and dose that more vacancies than interstitials are formed in the region of the crystal where high activation is required (near the surface). These vacancies stop the formation of boron clusters and make all the boron atoms electrically active. A fairly narrow window of implant energy and dose was used in order not to render the silicon surface amorphous. This window is smallest for carbon implants (instead of boron) and largest for germanium.

Czochralski-grown silicon typically contains $10^{18} / \mathrm{cm}^{2}$ oxygen from the silica crucible. The oxygen can affect electronic and other properties, and it is crucial to investigate the transport properties of the oxygen. In Symposium $\mathrm{V}$ on Advanced Silicon for 
the 21st Century, P. Wilshow (Oxford Univ., UK) described the application of a dislocation locking technique to investigate oxygen transport in Czochralski-grown silicon with different oxygen concentrations. Specimens containing well-defined arrays of dislocation half-loops were subjected to isothermal anneals of controlled duration during which oxygen diffused to the dislocations. The stress required to bring about dislocation motion was then measured. The behavior of the dislocation unlocking stress versus annealing time plot was found to have distinct regimes based on temperature. For all temperatures investigated, the unlocking stress initially rose approximately linearly before reaching a constant value. At high temperatures $\left(650-850^{\circ} \mathrm{C}\right)$, oxygen diffusivity was found to be in agreement with the established values, with an activation energy of $\sim 2.5 \mathrm{eV}$. At low temperatures $\left(350-650^{\circ} \mathrm{C}\right)$, oxygen transport was strongly enhanced, with an activation energy of $\sim 1.5 \mathrm{eV}$ due to a different species, the oxygen dimer, being responsible for transport. The dislocation locking technique was also used to study the effect of the concentration of shallow dopants on oxygen diffusion.

For further details on the research reported at the E-MRS/IUMRS-ICEM 2006 Spring Meeting, see the symposium summaries that follow. Meeting highlights and additional symposium summaries are also available online at www. emrs-strasbourg.com.

\section{Trends in Nanoscience}

Among the trends discussed in Symposium A on nanoscience-from materials to applications - researchers discussed discoveries in the areas of coatings, substrates, and nanoparticles and the influence of biosystems on materials research.

L.P. Biró (Research Inst. for Technical Physics and Materials Science, Hungary) described examples of photonic crystals in the biological world during a presentation on butterfly wings. Nanostructure, Biró said, gives rise to color on the wings. In the Cyanophyrs remus, found in South America, the blue color on the dorsal side of the wings consists of $5-10-\mu \mathrm{m}$ single crystals, while the green color on the ventral side is attributed to a polycrystalline structure. In the Albulina metallica, found in the Himalayas, on the other hand, the colors were obtained from polycrystalline structures, although with a somewhat different microstructure. These biological photonic crystals were found to be very efficient reflectors and showed amazing flexibility. The colors are physical properties and not pigments. The wings of butterflies have a hierarchical structure, with scales $(50 \mu \mathrm{m} \times 100 \mu \mathrm{m})$ that have ridges and cross-ribs on the micrometer scale that further contain $<100-\mathrm{nm}$ nanostructures. Subtle differences in the structures yield very different colors. Biró said that understanding the microstructure of butterfly wings could lead to photonic-crystal materials with interesting properties.

In the area of nanoscale coatings, K.-L. Choy (Univ. of Nottingham, UK) demonstrated a method of combining nanocomposite coatings and nanomultilayered coatings to form super-hard nanostructured coatings. She deposited nano-multilayered protective coatings of $\mathrm{TiN} / \mathrm{TiSiN}$ and $\mathrm{CrN} / \mathrm{CrSiN}$ by using closed-magnetic-field unbalanced dc magnetron sputter ion plating. The TiN/TiSiN coatings showed much higher static indentation hardness (40.2 GPa) than $\mathrm{CrN} / \mathrm{CrSiN}$ (30.9 GPa). The TiN/ TiSiN coatings also showed higher criticalfailure-force resistance and scratch fracture toughness as well as better wear resistance, whereas the $\mathrm{CrN} / \mathrm{CrSiN}$ coatings showed lower friction values.

H. Habermeier (Max Planck Inst., Germany) reported that perfect substrates are needed to grow perfect ceramics, but by the use of imperfect ones it is possible to gain control over strain and defects and to produce electronically active templates for piezoelectric, ferroelectric, and ferromagnetic materials. He called these "functional substrates." He discussed manganite substrates and their effect on colossal magnetoresistance. He showed how crystals polished just a degree or two off-axis could produce a stepped structure with nanoscale dimensions. By changing the terrace width in relation to the diffusion length of a deposited species, control of the process could be gained, he said.

Core-shell magnetic nanoparticles have been observed to self-assemble into chainlike structures. As reported by M. Bonini (Univ. of Florence, Italy), nanoparticles consisting of a magnetic core $\left(\gamma-\mathrm{Fe}_{2} \mathrm{O}_{3}\right)$ and an organic shell were prepared by thermal decomposition of an iron carbonyl complex in the presence of oleic acid. The nanoparticles were dispersed in cyclohexane to form a stable ferrofluid. These dispersions were investigated by means of small-angle scattering of $x$-rays and polarized neutrons. A 1:3 molar ratio of iron/oleic acid produced nearly monodisperse spherical nanoparticles, with a radius of the magnetic core of about $3.5 \mathrm{~nm}$. These nanoparticles were seen to self-assemble in chain-like structures, even in the absence of an applied magnetic field. Upon evaporation of the solvent on a solid substrate and under an external magnetic field, the suspension of
$\gamma-\mathrm{Fe}_{2} \mathrm{O}_{3}$ nanoparticles formed complex ordered patterns, such as spokes, spirals, and concentric rings.

\section{Field-Effect Devices}

Symposium B, From Strained Silicon to Nanotubes: Novel Channels for FieldEffect Devices, addressed channel and gate stack materials issues for highperformance complementary metal oxide semiconductor (CMOS) devices. The exponential increase in computer performance captured by Moore's law has largely been based on continued shrinking of the MOSFET. However, the silicon oxide or oxynitride gate insulator is now only 1-2 nm thick, resulting in unacceptably high gate leakage. To further increase chip performance, replacing the traditional channel in the bulk Si(100) surface plane with novel materials is an attractive option. Symposium B offered a broad view, from materials fundamentals to device characteristics, for the most important alternative channel options, such as strained silicon, semiconductoron-insulator, germanium, III-V semiconductors, semiconductor nanowires, and carbon nanotubes. Contact with related communities was fostered in joint sessions with Symposium L on Characterization of High-к Dielectric Materials and Symposium E on Science and Technology of Nanotubes and Nanowires.

Three sessions explored strained silicon. S. Thompson (Univ. of Florida, USA) reported that process-induced strain, achieved at low cost with stressed dielectric films covering the gate stack and with epitaxial SiGe source/drain regions, is a main device-level performance driver in current desktop processors. He predicted that further increases in strain will deliver nearly proportionally higher carrier mobilities. However, substrate-induced strain is still under consideration, and A.-T. Pham (Technische Univ. Braunschweig, Germany) outlined a new, fast Monte Carlo method to simulate biaxially strained Si/SiGe $p$-type FET devices. F. Houdellier (CEMES-CNRS, France) showed that two-dimensional strain and composition maps in SiGe with nanometer resolution can be recorded with electron holography, while T. Perova (Univ. of Dublin, Ireland) focused on micro-Raman spectroscopy for strain detection. Strained silicon-on-insulator was discussed by A. Lochtefeld (AmberWave Systems, USA), who described new ways to manufacture strained layers beyond the critical thickness. S. Takagi (MIRAI-AIST, Japan) shed light on a Ge condensation technique to fabricate dislocation-free strained SiGe-on-insulator and Ge-on- 
insulator, including novel approaches to create locally strained substrates.

For Ge and III-V semiconductors, the lack of high-quality gate dielectrics has been a main roadblock, and progress reports were particularly well-attended. M. Heyns and M. Caymax (IMEC, Belgium) and M. Fanciulli (MDM-CNR-INFM, Italy) reported that, contrary to conventional wisdom, interfacial $\mathrm{GeO}_{2}$ in high-permittivity gate stacks on Ge does not degrade the electrical properties, provided the appropriate oxidation state can be ensured. S. Koester (IBM, USA) and S. Oktyabrsky (State Univ. of New York at Albany, USA) evaluated Si interlayers between III-V semiconductors and high- $\kappa$ oxides as a step toward inversion-mode III-V MOSFETs. V.V. Afanas'ev (Katholieke Univ. Leuven, Belgium) and G. Seguini (MDM-CNR-INFM, Italy) employed internal photoemission spectroscopy to determine conduction-band offsets between high- $\kappa$ oxides and $\mathrm{Si}, \mathrm{Ge}$, and GaAs. Remarkably, for most oxide-semiconductor combinations, they found nearly identical conduction-band offsets. A simple attempt to predict band offsets based on charge-neutrality levels was described by J. Robertson (Cambridge Univ., UK).

M.V. Fischetti (Univ. of Massachusetts, USA) said that another potential roadblock for Ge and III-V channels is the "density of states bottleneck," degrading drive currents at short channel lengths. To circumvent this and other issues, several speakers postulated a fundamental redesign of the Ge and III-V MOS transistor. K. Saraswat and T. Krishnamohan (Stanford Univ., USA) described a doublegate heterostructure with a high-mobility Ge channel sandwiched between two Si layers, designed to reduce leakage. M. Passlack (Freescale, USA) described a paradigm shift away from inversionmode MOSFETs to "flatband-mode" devices based on undoped III-V channels. The performance potential of such "implant-free" III-V MOSFETs was studied by K. Kalna (Glasgow Univ., UK) using Monte Carlo simulations.

Rapid progress is being made in basic nanowire and nanotube device integration. Si and SiGe nanowire growth and early device demonstrations were discussed by U. Gösele (Max Planck Inst., Germany). L. Samuelson (Lund Univ., Sweden) gave a vivid overview of III-V semiconductor nanowires for photonics and electronics, and Y.-M. Lin (IBM, USA) discussed carbon nanotube transistors, demonstrating a ring oscillator built on a single nanotube. W.M. Weber (Qimonda, Germany) reported on shortchannel Si nanowire transistors with a NiSi source/drain.

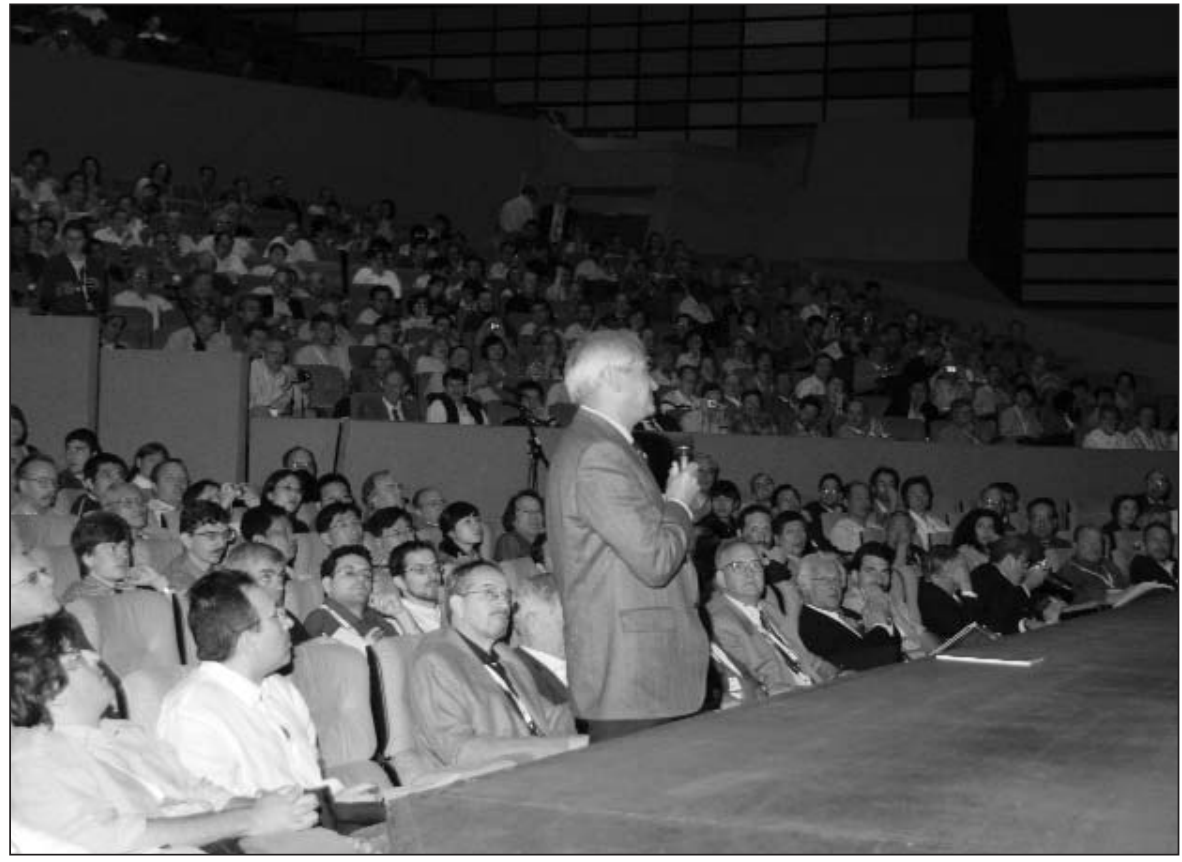

Attendees participated in a question and answer session during the plenary event.

\section{Si Nanocrystals}

Symposium C on Si nanocrystals for nanoelectronics and sensors covered the topics of silicon nanocrystal growth, characterization, modeling, simulation, theory, and properties. Applications in nanocrystal nonvolatile memory devices, photodetectors, light-emitting devices (LEDs), sensors (including biosensors), and molecular biology applications were discussed.

Highlights included results related to the fabrication of double layers or multilayers of Si nanocrystals within $\mathrm{SiO}_{2}$, ordering and self-assembly of nanocrystals in two-dimensional layers, and their application in novel nanoelectronic devices. Results on the improvement of nonvolatility by self-aligned double nanocrystal layers within the dielectric were demonstrated. The use of high- $\kappa$ dielectrics in the gate stack of the MOSFET memory structures was investigated by several presenters. Investigations on light emission from Er-doped Si nanoclusters and the luminescence quenching processes limiting quantum efficiency in these devices were presented.

Other highlights included surface functionalization of nanostructures for molecular biology applications and highconfinement nanophotonic devices for on-chip sensing. Applications of porous silicon as a nanostructured sensing material and in micromachining were investigated by different presenters.

\section{Si-Based Photonics}

Two of the major goals in silicon photonics are a silicon-compatible light source and, eventually, a silicon laser. There have been many approaches to achieving these goals, and recently, a Raman laser was demonstrated using Si. A more practicable approach will, however, be needed, and demonstrations of optical gain in $\mathrm{Si}$ nanocrystals have raised some hopes. These findings have been controversial and were heavily debated in Symposium D on Silicon-Based Photonics.

A new approach involving a nanopatterned Si surface was demonstrated. This yields infrared radiation, and optical gain appears to be confirmed, although the underlying mechanisms are still unknown. On the detector side, however, progress is more clear, and CMOScompatible Ge infrared detectors working well up to 10 Gbit were demonstrated.

P. Fauchet (Univ. of Rochester, USA) addressed the future of optical interconnects on silicon circuits. He said that the computer architect is the person who drives the use of technology. Data transfer across a chip is more important than transistor speed, and this transfer must be done at high speed with no distortion and cross-talk, and with low heat dissipation. Fauchet discussed four metrics: delay, bandwidth density, power delay product, and jitter. In Fauchet's discussion on the use of lasers on- and off-chip, 
the general conclusion was that off-chip lasers are currently the only solution until a silicon-based laser can be developed. It is likely that optical interconnects will not take over from copper interconnects for another 10 years at least.

\section{Nanotubes/Wires}

Symposium E on the Science and Technology of Nanotubes and Nanowires covered recent progress in the design, manufacture, and characterization of nanotubes and nanowires and the new developments that are leading to possible commercial applications of these materials in the near future.

Germanium nanowires are interesting for electronic applications because of quantum size effects and the fact that they are compatible with existing Si circuits. However, Ge nanowires oxidize easily, which degrades their electronic properties. E. Sutter (Brookhaven Natl. Lab, USA) described the formation of carbon graphene layers on the surfaces of $\mathrm{Ge}$ nanowires to prevent oxidation. She used in situ TEM observations to study the synthesis of the nanowires. The nanowires were grown using a Au catalyst at the tip, which was actually a Au-rich Au-Ge material. Dark Au spots were observed on the nanowires due to Au clusters migrating to the surface. While an oxide layer formed immediately, it was removed by heating to $200-300^{\circ} \mathrm{C}$. Ordered C-shells, in the form of graphene sheets, were formed on the nanowires due to the presence of the Au clusters on the surface; the residual $\mathrm{Au}$ on the surface triggered the formation of protective graphene layers on the surface. Sutter said that the technique could also be used for other semiconductor wires.

Gallium nitride nanowires offer exciting prospects for new devices if their growth can be controlled. L. Geelhaar (Infineon Technologies, Germany) described growth using plasma-assisted molecularbeam epitaxy (MBE) wherein the galliumto-nitrogen ratio was varied. With initial nitrogen-rich conditions, the nanowires grew to an upper limit of about $7 \mu \mathrm{m}$. Subsequent gallium-rich conditions thickened the wires in a controlled manner. The growth appears to be epitaxial and offers the prospect of heterostructures on a fine scale.

D. Keller (Univ. of Basel, Switzerland) reported that carbon nanotubes (CNTs) can be made into FETs that are very sensitive to the environment. Keller showed how benzene derivatives could change the transistor performance while alkyl silane could passivate the surface and poly(methyl methacrylate) (PMMA) could protect the contacts. In another presentation on CNTs, M. Fuhrer (Univ. of Maryland, USA) said that single- or multiwalled CNTs (>1 mm) can be formed into FETs by placing them on an oxidized silicon wafer and placing metal contacts on the CNT. These are Schottky barriers, and because they have high resistance, they tend to control the performance of the FET. Cycling the voltage on the transistor caused changes in the characteristics that could be reversed by exposing the device to air. It is believed that the air increases the $p$-type doping of the CNT and allows the contacts to perform better.

\section{Nanoscale Imaging and Metrology}

Symposium F on Nanoscale Imaging and Metrology of Devices and Innovative Materials addressed recent advances in imaging materials properties and structures at the nanoscale. P. Eyben (IMEC, Belgium) described how to use scanning spreading resistance microscopy (SSRM) to determine the junction position with an uncertainty of a few percent. F. Giannazzo (CNR-IMM, Italy) described the possibility of directly measuring carrier-drift mobility in nanostructures by using a combination of scanning capacitance microscopy (SCM) and SSRM.

T. Kelly (Imago Scientific Instruments, USA) used pulsed-laser-assisted sample atom emission on semiconductors to obtain a three-dimensional image of ultrashallow junctions as well as insulators. R. Wiesendanger (Univ. of Hamburg, Germany) illustrated nanoscale imaging by using a modified AFM. Wiesendanger presented extraordinary high-resolution imaging of magnetic properties down to single atoms.

M. Miles (Univ. of Bristol, UK) presented an alternative approach to high-speed AFM using resonance to scan the probe in the fast-scan direction. He showed one example of high-speed AFM of a chitosan (biological) film with a speed 3000 times that of conventional AFM, with no damage to the sample. In another example using near-field optical microscopy, polymer spherulites were scanned. Using the conventional technique, the speed was 1 frame in $1000 \mathrm{~s}$, whereas using resonance scanning, a speed of 120 frames/s was achieved.

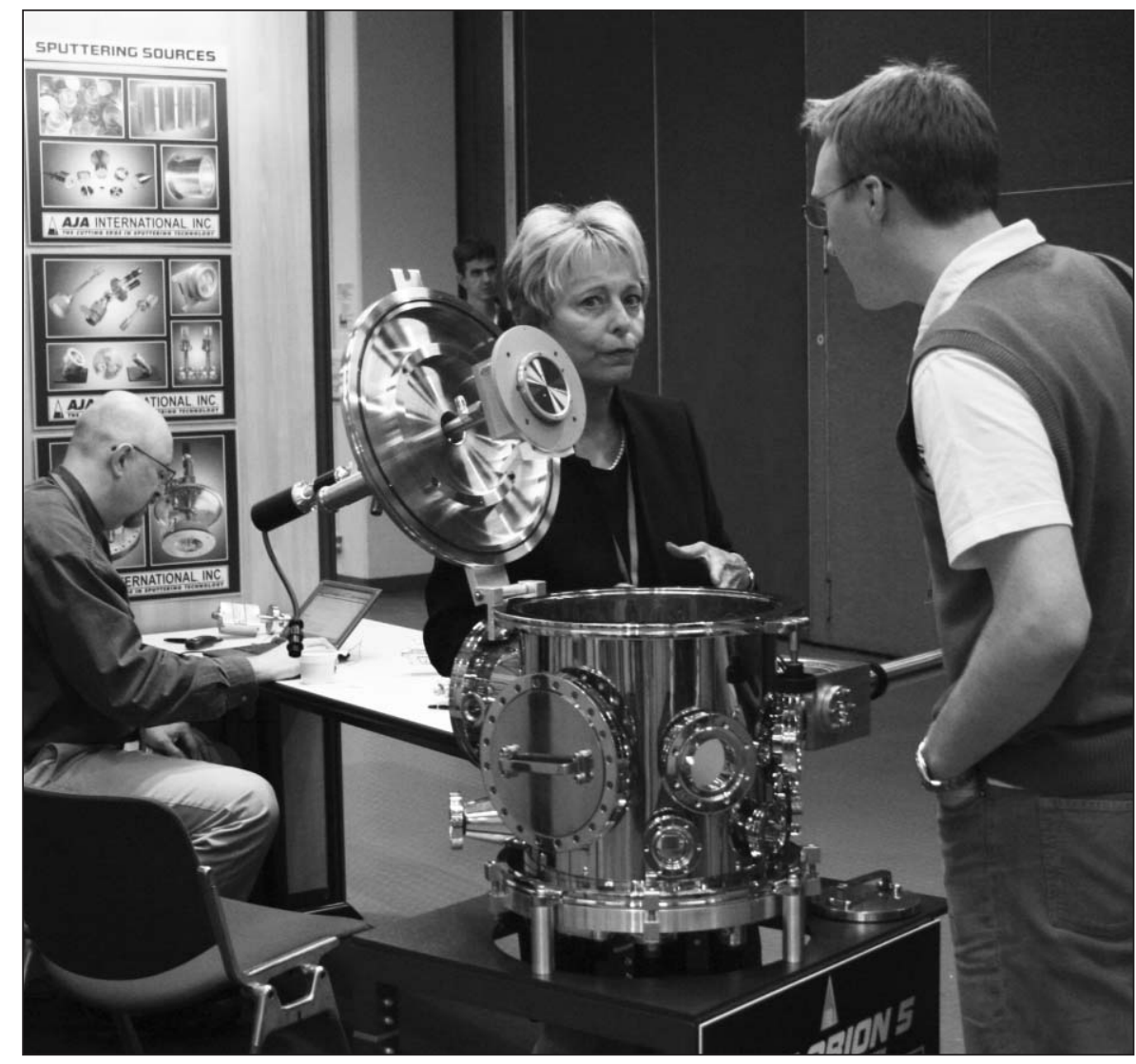

During the week-long meeting, participants had the opportunity to search for resources and products at the equipment exhibit. 
In the area of dielectrics, an AFM conductive tip was used to locally measure conduction through dielectrics, and nanometer lateral resolution was used to image the breakdown reliability of dielectrics. Other highlights included carrier profiling on semiconductors, the possibility of imaging stress and strain by TEM, and imaging nanotribology and nanomechanics.

\section{Micro- and Nanosystems}

In Symposium G addressing Functional Materials for Micro- and Nanosystems, topics included single-molecule detection and fabrication of nanodevices. P. Yang (Univ. of California, Berkeley, USA) described results by his group on using silica nanoparticles to detect the transport of single molecules such as DNA and proteins. There are two mechanisms at work, he said. First is the actual blockage, resulting in ionic current. However, at lower concentrations of the ionic medium in which the device operates (e.g., $\mathrm{KCl}$ ), there is also DNA-induced cation enrichment that leads to an increase in the current. Thus, there is a critical concentration between these two conditions. Yang said that the use of inorganic nanotubes / nanowires for single-molecule detection represents a new sensing platform and shows significant promise.

In another presentation, it was shown that $\mathrm{ZnO}$ is one of the most promising materials for fabricating nanodevices for gas sensors and for optoelectronic devices. S. Kim (Univ. of California, Davis, USA) presented a simple and effective method that allows $\mathrm{ZnO}$ nanowires to be selectively bridged between two prefabricated electrodes without using a metal catalyst or further complicated processes. This method allows for reproducible fabrication of a number of nanodevices based on individual or networked nanowires without using electron-beam lithography techniques. Kim described the technique as a "one-step thermal method." Previous studies on nanomaterials focused on individual nanowires selected from an original growth substrate, then wired with appropriate electrical contacts for electrical measurements.

\section{Large-Area Electronics}

In Symposium I on Thin Films for Large-Area Electronics, sessions on polycrystalline silicon focused on high-mobility thin-film transistors (TFTs) produced by laser annealing of amorphous silicon layers. Issues related to device stability and short-channel effects were discussed by M.-K. Han (Seoul Natl. Univ., Korea) and more advanced device architectures, including lightly doped drain and asym-

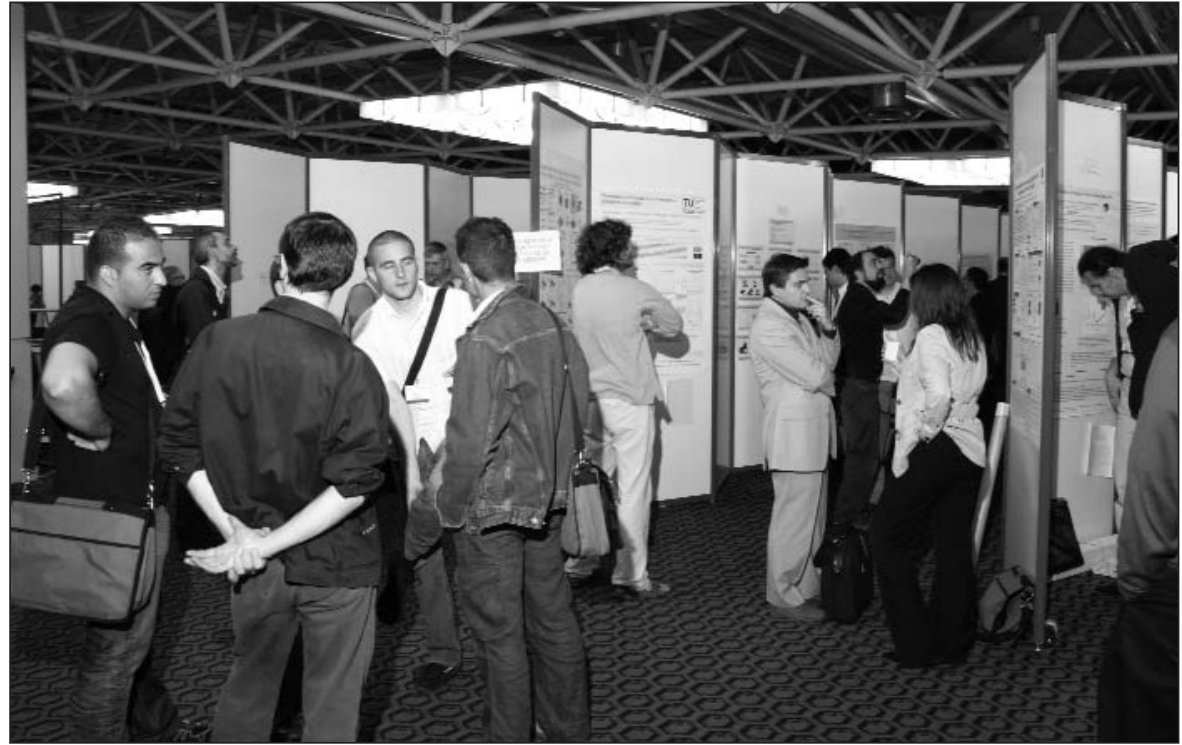

Meeting participants engaged in further discussions during the poster sessions.

metric fingered TFTs, were presented by several speakers. Low-temperature device processing on flexible substrates is showing increasing interest, and F. Templier (CEA-LETI, France) presented a polysilicon TFT active matrix fabricated on metal foils for organic LED applications. This was also the subject of a review by S. Wagner (Princeton Univ., USA) on flexible electronics, where mechanical properties of individual layers as well as their interaction with the rest of the sample are of concern.

Generally, high-quality polycrystalline silicon (poly-Si) must be produced by laser recrystallization of amorphous silicon. A. Nathan (Univ. of Waterloo, Canada) presented results on poly-Si TFTs directly grown by plasma-enhanced CVD (PECVD). They possessed very high carrier mobilities, for both electrons and holes, allowing for CMOS transistors to be grown from PECVD material. This result could have a significant impact on the large-area electronics industry.

Growth processes of thin films were addressed, with particular emphasis on micro- and polycrystalline silicon deposition at low temperature by plasma processes. N.-M. Hwang (Seoul Natl. Univ., Korea) emphasized the importance of charged clusters in thin-film deposition, which also form the basis for polymorphous and large-grain microcrystalline silicon deposition.

The wide range of thin-film characterization techniques was discussed. In particular, M. Vanecek (Academy of Sciences of the Czech Republic) presented Fourier transform photocurrent spectroscopy as a high-sensitivity technique to characterize sub-gap absorption in silicon, diamond, and organic thin films.

M. Schmidt (Hahn-Meitner Inst., Germany) reported solar cell efficiencies of up to $19 \%$ and discussed the prospect of attaining a $25 \%$ efficiency on heterojunction-based devices. In discussing imaging sensors, D. Caputo (Univ. of Rome "La Sapienza," Italy) described a-SiC:H-based devices which have a high UV efficiency. Micromachining applied to amorphous silicon thin films is emerging as an exciting alternative to well-established polycrystalline silicon devices. J. Conde (INESC MN, Portugal; Inst. Superior Técnico, Portugal) presented results on extremely high $Q$ factors for microresonators. The field of imaging and positionsensitive detectors is expanding quickly.

A.V. Kolobov (CNRS UMR, France; AIST, Japan) described phase-change effects in chalcogenides. These materials are important in both rewritable DVDs and future electronic nonvolatile memories to replace flash memory.

Organic semiconductors are experiencing a fast expansion. Fundamental aspects of the materials, such as the effect of processing on their optical properties as well as their application to thin-film transistors, were largely covered in the symposium.

\section{Nanoscale Functional Oxide Films}

J. Scott (Cambridge Univ., UK) opened Symposium J on Synthesis, Processing, and Characterization of Nanoscale Func- 
tional Oxide Films with a discussion of ferroelectric materials. He said that some behaviors, such as thickness-dependent ferroelectricity, surprisingly persist at the nanoscale, and that purely quantum effects such as the confinement of energy states are not observed. L. Pintilie (Max Planck Inst., Germany) and G. LeRhun (Max Planck Inst., Germany) said that in the majority of cases, the derived numerical values obtained from basic measurements and theoretical models do not take the microstructure into consideration. This produces a large scatter in the reported results for the same material, making it very difficult to distinguish between intrinsic and extrinsic properties of the material.

E. Garfunkel (Rutgers Univ., USA), A. Dimoulas (Univ. of Athens, Greece), and M. Frank (IBM, USA) focused on the thin high- $\kappa$ oxide materials. They presented the state of the art of this research for $\mathrm{Si}$, which is of prime importance for technical applications of the MOS transistors, as well as for various substrate materials such as Ge.

The formation of crystallographic phases in thin-film oxides is a very important phenomenon, directly related to the expected physical properties of the material. A. Kaul (Moscow State Univ., Russia) demonstrated the epitaxial stabilization of phases as a tool for synthesis of new thinfilm oxide materials.

S. Pennycook (Oak Ridge Natl. Lab, USA) illustrated that the aberration-corrected scanning TEM provides nanoscale depth resolution and that an individual Hf atom can be located within a $\mathrm{Si} / \mathrm{SiO}_{2} / \mathrm{HfO}_{2}$ gate dielectric structure with tri-dimensional precision of $0.1 \mathrm{~nm} \times 0.1 \mathrm{~nm} \times 0.1 \mathrm{~nm}$.

\section{Chalcogenide Materials Systems}

Symposium O on Thin-Film Chalcogenide Photovoltaic Materials saw strong participation from researchers in CdTe thin-film PV technology, whereas previous meetings on this topic have been dominated by CIGS thin-film technology. It is apparent that the two chalcogenide materials systems share common issues, such as similar approaches to meet device performance goals, analysis tools, characteristics, and device modules.

Research and development on CIGS thin films and devices has matured to the point that high-quality materials and highefficiency solar cells are achieved by different laboratories using different fabrication methods and a variety of buffer layers such as $\mathrm{CdS}, \mathrm{Zn}(\mathrm{S}, \mathrm{OH})$, and $\mathrm{In}_{2} \mathrm{~S}_{3}$. Potentially inexpensive deposition methods, such as electrodeposition, are making continual progress. Reaction pathways from different starting precursors are much clearer now than before, due to thorough investigations by sophisticated techniques, supported by calculations, of growth mechanisms of this multinary compound. The laboratory-scale device performance for this thin-film solar cell is almost $20 \%$.

In the area of CdTe technology, the cadmium life-cycle toxicity is much less than expected and is much lower than those of coal, fossil fuel, or PV silicon. Device performance has been stagnant, and enhancement is needed in the opencircuit voltage. Speakers addressed the influence of $\mathrm{Cu}$ in $\mathrm{CdTe}$ device performance and stability. Speakers also addressed whether the band structure of the device operates as an $n-p$ or $n-i-p$ device. The highest efficiency demonstrated to date is $16.5 \%$.

Several presentations centered on the power of bulk and surface characterization tools. Significant advances were discussed that are related to the chemistry at grain boundaries and interfaces as well as compositional and electronic inhomogeneities. It is critical to have a deeper understanding of how CIGS and CdTe-based devices operate. New classes of materials for the future generation of solar cells were introduced, along with their benefits.

Industry representatives for both CIGS and CdTe technologies described progress to date in terms of current production capacity and the addition of new capacity in the range of 15-75 MW. They also described scientific and technological challenges that must be overcome, which the community as a whole may want to address, to accelerate manufacturability and enhance the long-term stability of PV modules.

\section{Solid-State lonics}

In Symposium P on Solid-State Ionics: Mass and Charge Transport at Various Length Scales, a number of reports showed the occurrence of new and unexpected phenomena at the nanoscale due to the size reduction in thin films, heterostructures, composites, and nano-sized ceramics. Investigations with AFM tips on AgI nanoplates, for example, showed ionic conductivities that were orders of magnitude higher than in a standard polycrystalline material. Such nanoplates may be suitable in a new type of nanobattery. It became clear that nano-ionics is going to provide composite materials with enhanced storage capacities for batteries and new low-temperature electrolytes for micro fuel cells.

Due to the importance of mixed-ionic electronic conducting materials in fuel cells and permeation membranes, the transport properties of mixed conducting oxides are a focus of research. Innovative studies were reported, for example, on the mechanisms of oxygen transport and exchange kinetics at surfaces; a detailed analysis of surface diffusion of oxygen on $(\mathrm{La}, \mathrm{Sr}) \mathrm{MnO}_{3}$ was also presented. It was also reported that novel ion and mixed conducting glasses were developed for use in lithium-ion batteries.

Theory has become an important and indispensable tool for the development of materials with enhanced control of electronic and ionic transport in the bulk and at grain boundaries. Oxygen-ion conductors-in particular, ceria-derived compounds-are now well understood down to the atomic scale. The combined work using micro- and nanoanalysis and theory on ferroelectrics allows refined control of piezoelectric actuators and corresponding information storage materials.

Important progress has also been made in spectroscopic techniques such as nuclear magnetic resonance with respect to an atomistic understanding of ion transport. The results are now an important tool for the improvement of battery materials. Photoelectron spectroscopy typically carried out under UHV conditions has recently been made available for measurements under elevated pressure. This technical progress opens ways to study surfaces under realistic pressure conditions.

\section{Chemical and Bio-Sensing Transistors}

Symposium Q addressed Chemical and Bio-Sensing Transistors: From Materials to Systems. ZnO as a wide-bandgap material for invisible sensors was presented by R. Yakimova (Linköping Univ., Sweden). M. Eickhoff (Munich Univ. of Technology, Germany) discussed GaN/AlGaN/GaN FETs for detection of penicillin. A.R Phani (Univ. of L'Aquila, Italy) reported that $\mathrm{WO}_{3}$ nanowires as sensing materials collapse at $500^{\circ} \mathrm{C}$ but perform well at $300^{\circ} \mathrm{C}$. A. Georgakilas (Univ. of Crete, Greece) had made a study of the parameters of $\mathrm{AlGaN} / \mathrm{GaN} \mathrm{pH}$ sensors. P. Gouma (State Univ. of New York, USA) used nanowires with controlled crystal structure to obtain selective chemosensors. Isoprene in the breath could be detected as a marker of cholesterol.

In the area of functionalized sensors, A. Spiegel (Johannes Kepler Univ., Austria) described an organic FET used for interfacing with living cells. P. Pacher (Graz Univ. of Technology, Austria) used an IR method to reveal the reactive dipoles on his sensor surfaces. Y. Alifragis (Univ. of Crete, Greece) reported the use of AlGaN/ 
GaN FET sensors with, for example, a change of $-52.4 \mathrm{mV} /$ decade for $\mathrm{K}^{+}$ions. K. Shimanoe (Kyushu Univ., Japan) presented a study of the necessary optimization of the parameters of a FET sensor for $\mathrm{NO}_{2}$ in order to realize the commercialization of the device.

In the area of organic transistors, G. Malliaras (Cornell Univ., USA) reviewed contributions on conducting polymer transistors for chemical and biological sensors, while G.M. Farinola (Univ. of Bari, Italy) showed how biofunctionalization of conjugated backbones results in high-performance enantioselective TFTs. M. Berggren (Linköping Univ., Sweden) discussed polythiophene-based electrochemical transistors in sensor and actuator applications.

\section{Wide-Bandgap Semiconductors}

Symposium S on Materials Science and Technology of Wide-Bandgap Semiconductors featured several highlights. A brief overview on the limitations and potentials of a number of different approaches to growing bulk and thick films of $\mathrm{GaN}$ was presented. Hydrothermal and ammonothermal techniques, which have been successfully used to grow $\mathrm{ZnO}$ and quartz, respectively, were proposed as potential approaches to produce large-area, highquality GaN substrates. The properties of state-of-the-art bulk $4 \mathrm{H}-\mathrm{SiC}$ and the deposition of electronic-grade homoepitaxial layers were presented. In addition, the fabrication and characteristics of recently commercialized high-power, highfrequency devices were discussed.

The growth and the properties of 2-in.diameter, 2-mm-thick freestanding $\mathrm{GaN}$ substrates grown by hydride vapor-phase epitaxy (HVPE) in a vertical hot-wall reactor at atmospheric pressure were presented. Although ammonothermal and molten solution techniques have great potential, the samples currently produced by these methods are still very small as a result of the lack of optimization of nutrient transport, mineralizer, and temperature gradients. This problem was addressed by using 2-in.-diameter metalorganic CVD (MOCVD) GaN/sapphire templates as seeds to grow largearea GaN by a low-pressure solution technique. Luminescence and TEM studies demonstrated improved optical and structural properties of these homoepitaxial films relative to the properties of the original MOCVD template. GaN templates produced by laser lift-off and wafer bonding were proposed as an option for low-cost/large-area GaN substrate fabrication. The quality and functionality of the bonded $\mathrm{GaN}$ on a $\mathrm{SiO}_{2} /$
Si(100) substrate was evaluated by luminescence and Raman spectroscopies and by LED fabrication.

Nonpolar GaN has been recently proposed as an ideal substrate to avoid problems introduced by spontaneous polarization in nitrides. Native defects controlling the electrical properties of $a$-plane GaN films were investigated by positron annihilation spectroscopy. A detailed description of the structural properties of $10-\mu m$-thick InN films deposited on (0001) sapphire substrates by plasma-source MBE was presented. Despite the high concentration of carriers, typically $1.2 \times 10^{18} / \mathrm{cm}^{2}$, these films exhibited Hall mobilities of 1500 $\mathrm{cm}^{2} / \mathrm{V} \mathrm{s}$ at room temperature.

The $a$-plane and $r$-plane GaN layers possess a large concentration of extended defects, which may preclude their potential application in high-efficiency LEDs. The role of deep levels on the electrical properties of GaN films was addressed using conventional deep-level transient spectroscopy and transient scanning capacitance microscopy. The lifetime of photoinduced carriers and its dependence on different decay mechanisms were investigated in GaN films.

The characterization of structural defects in $\mathrm{GaN}$ and $\mathrm{SiC}$ using selective etching in combination with TEM and Raman and cathodoluminescence spectroscopies was presented. Molten bases, hot acids, and photoelectrochemical etching processes were developed to reveal dislocations, inversion domains, and nano- and micropipes. Studies of the carrier diffusion length were carried out in as-grown and Si-doped $\mathrm{GaN}$ using cathodoluminescence measurements in a high-resolution scanning electron microscope. A recombination process involving donors and acceptors yielded the hole diffusion length.

The market value and opportunities for wide-bandgap semiconductor materials were discussed. It was noted that the optical and electronic properties of MOCVD AlN films deposited on Si substrates are strongly dependent on changes in the growth conditions from Al-rich to N-rich, which results in the incorporation of different defects that control the films' properties. Deposition and properties of AlGaN/ GaN 2DEG heterostructures grown on Fe-doped GaN substrates by HVPE techniques were addressed. The elimination of parasitic parallel conductance, combined with high carrier concentration and mobility, was reproducibly achieved, demonstrating that high-performance highelectron-mobility transistors (HEMTs) can be fabricated on GaN:Fe substrates.

Although considerable resources had been previously invested to control macro- scopic defects such as polytype inclusions, low-angle grain boundaries, and micropipes in $\mathrm{SiC}$, currently it is commonly accepted that dislocations are the main reason for the degradation of power devices, and they must be eliminated. It was also emphasized that reducing the thermoelastic stress in the growing crystal results in substrate improvement. Another highlight of this symposium was the demonstration that a freestanding HVPE $\mathrm{GaN}$ substrate with a dislocation density lower than $10^{5} / \mathrm{cm}^{2}$ has a thermal conductivity of about $230 \mathrm{~W} / \mathrm{K} \mathrm{m}$. This result is very important for the realization of high-power devices based on nitride semiconductors. Calculations based on kinetic Monte Carlo approximations provided an estimate of the mobility of nitrogen vacancies in $n$-type $\mathrm{GaN}$ and $\mathrm{InGaN}$ and the energy formation of nitrogen vacancy clusters.

The properties of GaN-based devices fabricated on a thin $\mathrm{Si}$ wafer bonded to polycrystalline $\mathrm{SiC}$ templates were discussed. This approach provides a largearea, low-cost substrate with good thermal conductivity. High-frequency devices fabricated on these templates show similar performance to those fabricated on Si substrates. AlGaN/GaN HEMTs directly fabricated on $\mathrm{Si}(100)$ substrates exhibit a sheet carrier density of $7.1 \times 10^{12} / \mathrm{cm}^{2}$ and a Hall mobility of $1500 \mathrm{~cm}^{2} / \mathrm{V} \mathrm{s}$ at room temperature. The preparation of nitride substrates with epi-ready surface quality was investigated for $\mathrm{N}$-face $\mathrm{GaN}$, resulting in reduced surface roughness. A combination of low-pressure, temperature, and gas atmosphere was required to achieve isotropic etching.

The needs of specific processing approaches for $\mathrm{SiC}$, different from that of the well-established Si technology, were emphasized. State-of-the-art SiC Schottky diodes and the industrial and commercial aspects of SiC-based devices were overviewed. Specific aspects of SiC device technology such as reactive ion etching and high barrier characteristics were also objects of discussions. Vertical Schottky diode structures with interdigitated semitransparent contacts were proposed in order to realize a high quantum efficiency UV detector.

In the area of GaN-based device fabrication and characterization, a novel UV detector structure based on GaN membranes was discussed. Detailed x-ray diffraction studies of InGaN films indicated that reported changes in the emission, which were previously attributed to compositional fluctuation arising from phase separation, result from changes in the growth mode from planar two-dimen- 
sional to three-dimensional (StranskiKrastanov transition), due to a strong, spatially varying strain profile.

\section{Advanced Microelectronic Devices}

As classical scaling of CMOS devices is slowing down, being progressively replaced by "performance engineering," new approaches are becoming the object of more and more intense research, such as strain engineering, new materials, and advanced annealing methods. The topics presented in Symposium U on Si-Based Materials for Advanced Microelectronic Devices: Synthesis, Defects, and Diffusion included new materials such as strained $\mathrm{Si}, \mathrm{Si}_{1-x} \mathrm{Ge}_{x}$, and issues such as defects, diffusion, ion implantation, and synthesis. Mainly, however, research was presented on ultrashallow junctions for future Si CMOS devices, spanning from the investigation of new methods for their formation to the fundamental understanding of dopants and defects.

T. Feudel (AMD, Germany), in his talk reflecting the increasing role of the performance-engineering approach for future CMOS devices, discussed the use of stress-enhancing methods to increase channel mobility and the potential of different techniques for the CMOS nodes such as stressed liner and spacer materials, strained silicon substrates, embedded $\mathrm{Si}_{1-x} \mathrm{Ge}_{x}$ and $\mathrm{Si}_{1-x} \mathrm{Gex}$ layers, different silicides, source/ drain pre-amorphization, and compatibility with flash lamp annealing.

Among the different advanced annealing techniques proposed for future ultrashallow junction formation, the potentialities of millisecond annealing with flashassist rapid thermal processing (fRTP) were discussed by J. Gelpey (Mattson, Canada). He showed that millisecond annealing with fRTP can deliver shallow, highly active, low-leakage $p$ - and $n$-type junctions meeting the 45 -nm requirements of the International Technology Roadmap for Semiconductors and beyond.

The physics involved in ultrashallow junction formation was largely discussed in the symposium. Pre-amorphization and subsequent solid-phase epitaxy regrowth, B co-implantation with $\mathrm{C}$ or $\mathrm{F}$, and millisecond annealing were addressed. The complex relationships existing between defect formation and evolution and B diffusion and activation were discussed by several speakers, including L. Pelaz (Univ. of Valladolid, Spain) and C. Fuccio (CEMES/CNRS, France).

The understanding of B clustering is a crucial challenge for ultrashallow junction formation that demands new experimental methods to investigate the microscopic structure of the agglomerates. This issue

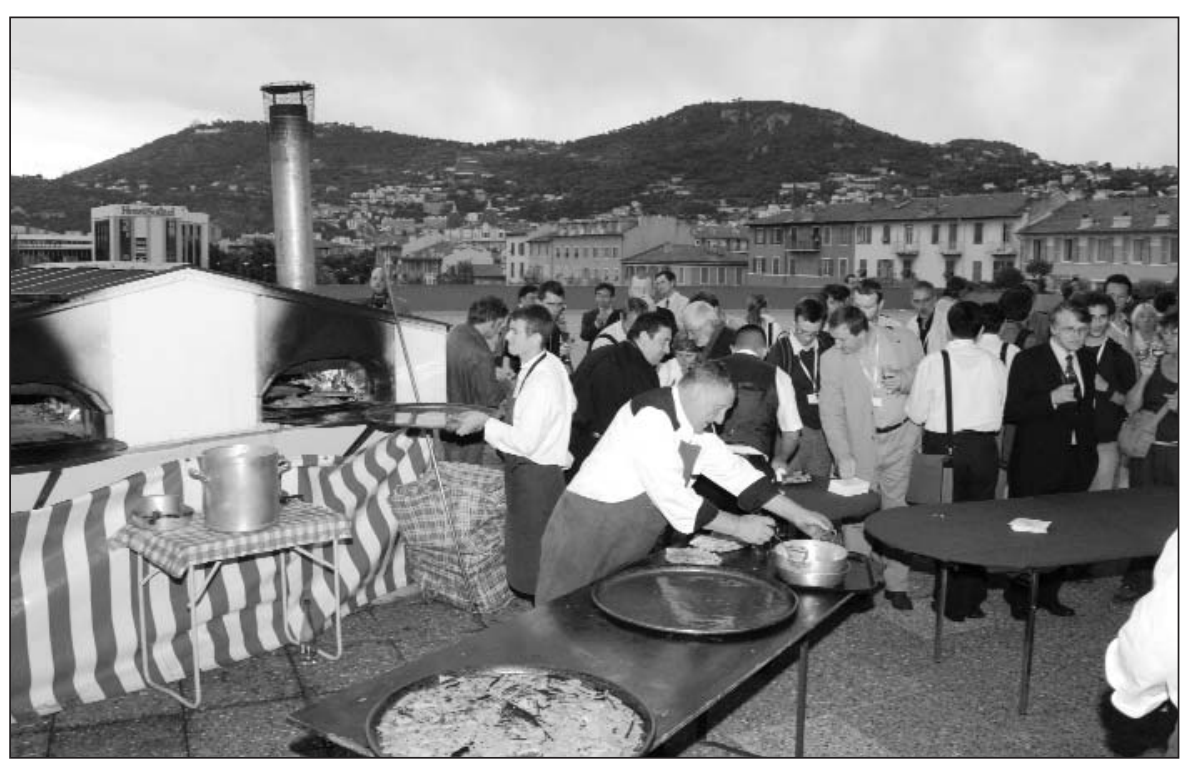

A banquet was held for the Meeting attendees.

was discussed in two presentations where B clustering at extremely high concentrations was studied by ion channeling, as shown by L. Romano (MATIS CNR-INFM, Italy) and by in situ high-resolution x-ray diffraction, as shown by G. Bisognin (MATIS CNR-INFM, Italy).

H. Bracht (Univ. of Muenster, Germany) showed that by combining experiments with isotopically controlled semiconductor multilayers and multiparameter continuum modeling based on numerical solutions of diffusion kinetic equations, it is possible to extract fundamental parameters describing self- and dopant-diffusion and obtain information on the nature, charge states, and energy levels of the point defects involved. A different approach was followed by D. De Salvador (MATIS CNR-INFM, Italy), who performed an experiment focused on direct measurement of the parameters governing the $\mathrm{B}$ diffusion as a function of the Fermi level. De Salvador proposed a set of simple reactions fully describing the microscopic mechanism of B diffusion involving differently charged point defects and interactions with the free holes.

Because the precise control of point defects is becoming more and more stringent for ultrashallow junction formation, a session of the symposium was dedicated to nonequilibrium phenomena and pointdefect engineering. Vacancy engineering seems to be a very promising method for annihilating the self-interstitials responsible for the unwanted phenomena of transient-enhanced diffusion and B clus- tering. Several methods were proposed for the introduction of vacancies in the material: N. Cowern (Univ. of Surrey, $\mathrm{UK}$ ) proposed vacancy engineering by $\mathrm{Si}$ ion implantation; vacancies can also be introduced in Si by epitaxially regrowing a pre-amorphized Si layer implanted with F, as extensively described by G. Impellizzeri (MATIS CNR-INFM, Italy) and theoretically investigated by V. Fiorentini (SLACS CNR-INFM, Italy).

Technology computer-aided design (TCAD) is an indispensable tool for the development and optimization of a new generation of electronic devices in industrial environments. P. Pichler (IISB, Germany) gave a comprehensive historical overview of the important results obtained during the last few years within the framework of European Commission projects, such as the clarification and full calibration of the agglomeration of selfinterstitials from small clusters to large loops, the investigation of the interactions of intrinsic point defects with dopants and impurities like fluorine and carbon, and major contributions to diffusion in silicon germanium alloys.

J. Slotte (Helsinki Univ., Finland) gave a broad overview of the capability of positron annihilation spectroscopy and showed that the dominant defect in $n$-type SiGe layers after proton irradiation is the E-center, the vacancy-phosphorus pair, which is mobile and pairs with a Ge atom during annealing. W. Vandervorst (IMEC, Belgium) showed that accurate and reliable characterization of carrier depth pro- 
files for ultrashallow junctions for future sub-45-nm CMOS technologies can be obtained with the microscopic four-point probe technique. Quantitative, direct mapping of continuous strain and composition distributions in strained $\mathrm{Si} / \mathrm{Ge}$ multilayers are obtainable by combining geometric phase analysis of high-resolution TEM images and electron holography, as shown by N. Cherkashin (CEMES-CNRS, France).

Two sessions were devoted to defects and impurities. A fundamental understanding of the electronic properties of the most abundant point defects and impurities is needed for good control of SiGe-based device performance. A. Mesli (CNRS/ULP, France) discussed how the Ge composition influences the deep levels, the allowed bands, and the diffusion mechanism of highly mobile defects. Many talks were devoted to hydrogenrelated or vacancy-related defects, such as the $\mathrm{As}_{2} \mathrm{~V}$ complex in silicon presented by H. Kortegaard Nielsen (Univ. of Aarhus, Denmark).

\section{MSE Education}

Symposium W on Education in Materials Science, Technology, and Engineering featured presentations and forum discussions that explored major issues facing materials educators worldwide and reported on innovative approaches to effective teaching at all levels.

One major issue of current universal interest is the introduction of nanotechnology into the fabric of formal academic programs. What should such programs cover? What are the merits of a dedicated degree program in nanotechnology as compared with the integration of nanoscience topics into the existing crowded materials science and engineering (MSE) curriculum? Does the introduction of nanoscience belong in $\mathrm{K}-12$, undergraduate, or primarily graduate level programs? Who can afford access to the required expensive instrumentation?

Participants heard numerous positive and encouraging accounts of recently initiated or proposed nanotechnology programs or curriculum additions in several regions, including the United States, Europe, Asia, and Central and South America, and accounts were presented of extraordinary funding for nano-education and research from government and industrial sources in many countries.

While the education of MSE students in nanotechnology is being widely hailed as the key to competitive national economic success in terms of preparation of the workforce, questions also arose about other fast-moving emerging fields that are of vital interest for academic and industrial research and education. Biomaterials and biomedicine, ecomaterials, sustainable processing and design of materials, and advanced energy-resource technologies are recognized as critical areas for developing national and global professional expertise and infrastructure in research and in industrial production. Discussions of how to keep curricula current and effective in all such evolving areas displayed a broadly held view that formal introduction of these topics may often be best achieved through specialized courses at the early graduate level or in focused continuing-education programs. Special success was reported from group project assignments that mixed students from different topical backgrounds; sharing a common task facilitated the effective exchange of knowledge.
Newly developed resources, software, and course materials were described and appraised, including online approaches for materials education, modules available for teaching and advanced computer simulations, and new approaches for capturing the all-important interest and excitement of students.

In many developing countries, progress is robust but inhibited in some instances by issues of economics and custom. Clearly, there is considerable scope for international cooperation in which students and faculty in advanced nations could collaborate in materials education and research and share ideas and resources while benefiting from both cultural and economic interactions. During a panel discussion on "Mega-Trend Challenges for Education in the Coming Decade," it was evident that the globalization of commerce and communications will open broader educational opportunities. However, the point was made that "traditional" academic structures and expectations will need to adjust in order to foster approaches that broaden and integrate programs, departments, and faculty to deliver the most relevant educational experiences for students.

One notable developing program of international collaboration, the Global School for Advanced Studies, was the focus of a special workshop session. This program will initiate collaborations in research and teaching on an international scale, with the vital goal of preparing outstanding future leaders in the field of materials research and education, whose perspectives are global and their experience broad.

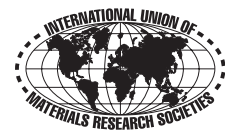

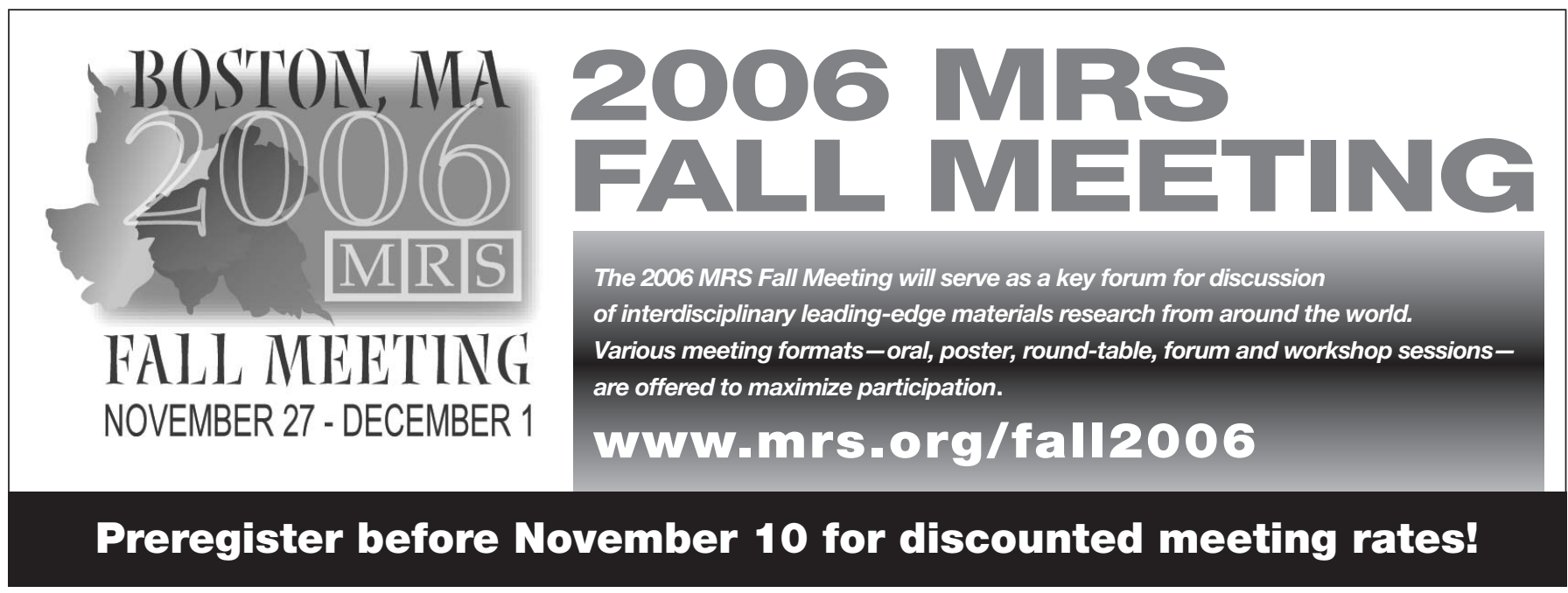




\section{E-MRS Announces New Opportunities for Its Members}

The executive committee of the European Materials Research Society (E-MRS) has made the decision to diversify and extend the activities of the Society beyond the organization of the E-MRS Spring and Fall Meetings. The Society's three new initiatives are books, short courses, and workshops.

E-MRS seeks authors and editors interested in preparing books on topics such as materials science, materials engineering, and materials processing. The books will be published under the framework of the Elsevier/E-MRS publishing agreement related to a monograph series of books titled the "E-MRS Monograph Work." Based on the contents of books in the monograph series, E-MRS plans to develop short courses to be held in conjunction with its Spring and Fall Meetings.

For its third initiative, E-MRS plans to establish Advanced Research Workshops on new topics in order to strengthen and develop relationships among scientists from various European countries. The Society will seek sponsorship from research agencies such as the European Science Foundation and NATO. The goal of these workshops is to initiate research activities in Europe and prepare the ground for future collaborative research programs and scientific networks.

For more information, contact E-MRS at 23 Rue du Loess, 67037 BP 20, Strasbourg Cedex 02, France; tel. 33-(0)3-88-10-63-72; fax 33-(0)3-88-10-62-93; e-mail emrs@emrs. c-strasbourg.fr; or access Web site www. emrs-strasbourg.com.

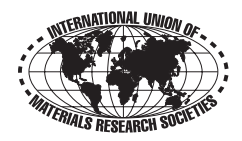

